RETRACTION

doi:10.1038/nature04183

\section{Formation of zirconium metallic glass}

Jianzhong Zhang \& Yusheng Zhao

Nature 430, 332-335 (2004)

In this Letter, we concluded, primarily on the basis of energydispersive X-ray diffraction, that metallic glass could be formed in pure zirconium under high pressure and temperature conditions. However, careful observations using an angular-dispersive method and imaging-plate detector, together with X-ray-transparent anvils (H. Saitoh, T. Hattori, H. Kaneko, Y. Okajima and W. Utsumi, unpublished results), have revealed that our conclusion was in error. We are now convinced that the disappearance of diffraction lines of zirconium observed in our energy-dispersive experiments should be interpreted instead as rapid crystal growth at temperatures above that of the $\omega-\beta$ phase transformation.

Our original misinterpretation was partly due to our assumption that significant crystalline growth could not occur at temperatures of less than one-third of the melting point of zirconium. To a greater extent, it was a result of the limitations of the experimental techniques then available, which only allowed viewing of a very narrow window of Debye diffraction rings and therefore increased the probability of missing the Bragg spots. The state-of-the-art techniques used by Saitoh et al. at the Synchrotron Radiation Research Center, Japan Atomic Energy Institute, Hyogo, are superior for highpressure research on non-crystalline materials.

We thank our Japanese colleagues for their careful work and for inviting us to observe their high pressure and temperature synchrotron X-ray diffraction experiments at beamline BL14B1 of SPring-8.

RETRACTION

doi:10.1038/nature04182

\section{Negative lattice expansion from the superconductivity-antiferromagnetism crossover in ruthenium copper oxides}

\author{
A. C. Mclaughlin, F. Sher \& J. P. Attfield
}

Nature 436, 829-832 (2005)

This Letter described several notable physical properties for lowdoped ruthenium copper oxides, from which conclusions concerning superconductivity in copper oxides were drawn. A key result was the observation of an unusual, negative, expansion of the lattice parameters and volume at temperatures below the $\mathrm{Ru}$ spin-ordering transition, $T_{\mathrm{Ru}}$, as shown in Figure 3. Unfortunately, we have discovered that a discrepancy in the algorithm for fitting the diffraction data led to erroneous shifts in the cell parameters and resulted in the apparent negative expansion. We retract the claim of negative lattice expansion and the consequent inferences concerning the existence of two states in the pseudogap regime of the copper oxide electronic phase diagram. However, an anomalous expansion of the separation between copper oxide planes below $T_{\mathrm{Ru}}$, as shown in Figure 3b, is reproducible and has subsequently been observed in other ruthenium copper oxide samples. Our results concerning large magnetoresistances and successive $\mathrm{Ru}$ and $\mathrm{Cu}$ spin-ordering transitions are not affected, so Figures 1 and 2 and the Supplementary Figures remain valid.

We thank S. Kimber and A. Williams for undertaking further calculations to determine the nature of the fitting problem.

\section{RETRACTION}

\section{doi:10.1038/nature04181 \\ RNA-interference-directed chromatin modification coupled to RNA polymerase II transcription}

Vera Schramke, Daniel M. Sheedy, Ahmet M. Denli, Carolina Bonila, Karl Ekwall, Gregory J. Hannon \& Robin C. Allshire

Nature 435, 1275-1279 (2005)

An earlier paper $^{1}$ by two of us, in which it was concluded that expression of a hairpin RNA homologous to ura4 RNA in Schizosaccharomyces pombe results in the production of siRNAs that bring about methylation of histone $\mathrm{H} 3$ on lysine 9 and recruitment of Swi6 to the ura4 gene, has been retracted ${ }^{2}$. This is because we are unable to reproduce the experiments involving expression of hairpin ura4 RNA because the plasmid sh-ura4SE-280, or fission yeast strains that contain it, do not exist. Attempts to reproduce these observations with other ura4-hairpin constructs have failed.

As a result, we cannot verify the observations reported in our Letter to Nature and shown in Figures 1,2 and 4a (lower panel) because these utilize the same plasmid sh-ura4-280 hairpin construct. We therefore retract the conclusions that transcription of an siRNA homologous target is required to bring about silent chromatin assembly, that transcription of an siRNA homologous target by T7 is not sufficient to bring about silent chromatin assembly, and that transcription of an siRNA homologous target is required to allow association of Agol with that target.

However, we have confirmed that truncation of the RNApol II CTD (removal of 17/28 heptad repeats) does alleviate silencing at centromeres, that centromeric siRNAs remain detectable (Figure 3), and that RNA pol II and Agol co-immunoprecipitate. In addition, the expression-profiling data generated for $r p b 1-11$ are robust.

V.S. has declined to sign this retraction as she maintains that the original conclusions are correct.

1. Schramke, V. \& Allshire, R. C. Hairpin RNAs and retrotransposon LTRs effect RNAi and chromatin-based gene silencing. Science 301, 1069-1074 (2003).

2. Schramke, V. \& Allshire, R. C. Retraction. Science 310, 49 (2005). 